\title{
Conditional Density Models for Asset Pricing
}

\author{
Damir Filipović*, Lane P. Hughston ${ }^{\dagger} \&$ Andrea Macrina $\ddagger \S$
}

* Swiss Finance Institute, Ecole Polytechnique Fédérale de Lausanne, Switzerland
$\dagger$ Department of Mathematics, Imperial College London, London SW7 2AZ, UK
$\ddagger$ Department of Mathematics, King's College London, London WC2R 2LS, UK
$\S$ Institute of Economic Research, Kyoto University, Kyoto 606-8501, Japan

\begin{abstract}
We model the dynamics of asset prices and associated derivatives by consideration of the dynamics of the conditional probability density process for the value of an asset at some specified time in the future. In the case where the price process is driven by Brownian motion, an associated "master equation" for the dynamics of the conditional probability density is derived and expressed in integral form. By a "model" for the conditional density process we mean a solution to the master equation along with the specification of (a) the initial density, and (b) the volatility structure of the density. The volatility structure is assumed at any time and for each value of the argument of the density to be a functional of the history of the density up to that time. In practice one specifies the functional modulo sufficient parametric freedom to allow for the input of additional option data apart from that implicit in the initial density. The scheme is sufficiently flexible to allow for the input of various types of data depending on the nature of the options market and the class of valuation problem being undertaken. Various examples are studied in detail, with exact solutions provided in some cases.
\end{abstract}

9 November 2011

Classification: C60, C63, G12, G13.

Key words: volatility surface, option pricing; implied volatility; Bachelier model; information-based asset pricing; nonlinear filtering; Breeden-Litzenberger equation.

\section{Introduction}

This paper is concerned with modelling the dynamics of the volatility surface. The problem is of great practical interest to traders, and as a consequence has an extensive mathematical literature associated with it. In this brief report, we shall not attempt to review earlier work in the area, but refer the reader, for example, to Schönbucher (1999), Gatheral (2006), Schweizer \& Wissel (2008a,b), Carmona \& Nadtochiy (2009, 2011), and references therein. 
Put informally, the general idea of the paper is as follows. We fix a numeraire, and write $\left\{A_{t}\right\}$ for the value process of some tradable financial asset expressed in units of that numeraire. We fix a time $T$ and assume that no dividends are paid from time 0 up to $T$. Letting $\mathbb{Q}$ denote the martingale measure associated with the chosen numeraire, we have

$$
A_{t}=\mathbb{E}^{\mathbb{Q}}\left[A_{T} \mid \mathcal{F}_{t}\right],
$$

for $t \leq T$, and

$$
C_{t T}(K)=\mathbb{E}^{\mathbb{Q}}\left[\left(A_{T}-K\right)^{+} \mid \mathcal{F}_{t}\right],
$$

where $C_{t T}(K)$ denotes the price at time $t$ of a $T$-maturity call option with strike $K$. The associated conditional density process $\left\{f_{t}(x)\right\}$ for the random variable $A_{T}$ is defined by

$$
\int_{-\infty}^{y} f_{t}(x) \mathrm{d} x=\mathbb{E}^{\mathbb{Q}}\left[\mathbb{1}\left\{y>A_{T}\right\} \mid \mathcal{F}_{t}\right]
$$

Then for the asset price we have

$$
A_{t}=\int_{\mathbb{R}} x f_{t}(x) \mathrm{d} x
$$

and the corresponding option prices are given by

$$
C_{t T}(K)=\int_{\mathbb{R}}(x-K)^{+} f_{t}(x) \mathrm{d} x .
$$

Instead of modelling $\left\{A_{t}\right\}$ and then determining $\left\{C_{t T}(K)\right\}$, our strategy is to model the conditional density process. Then the underlying asset price process and the associated option prices are determined by (1.4) and (1.5). Roughly speaking, the idea is to model $\left\{f_{t}(x)\right\}$ in such a way that it contains some parametric freedom that can be calibrated to a specified range of initial option prices. Models for conditional densities have been considered in various contexts in finance. These include for example applications to interest rates (Brody \& Hughston 2001a,b, 2002, Filipović et al. 2010), and to credit risk (El Karoui et al. 2010). Although mostly different from what has previously appeared in the literature, our approach to modelling the volatility surface is similar in spirit in some respects to that of Davis (2004).

Let us consider in more detail the class of assets that will form the basis of our investigation. We introduce a probability space $(\Omega, \mathcal{F}, \mathbb{P})$ with filtration $\left\{\mathcal{F}_{t}\right\}_{t \geq 0}$, where $\mathbb{P}$ is the "physical" measure and $\left\{\mathcal{F}_{t}\right\}$ is the market filtration. We assume that price processes are adapted to $\left\{\mathcal{F}_{t}\right\}$. We assume the absence of arbitrage, and the existence of an established pricing kernel $\left\{\pi_{t}\right\}$ associated with some choice of base currency as numeraire. We work in the setting of a multi-asset market, and do not assume that the market is complete.

We write $\left\{A_{t}^{i}\right\}_{i=0,1, \ldots, N}$ for the price processes of a collection of non-dividend-paying tradable financial assets. Prices are expressed in units of the base currency. We refer to asset $i$ as $A^{i}$. We model the $\left\{A_{t}^{i}\right\}$ as Ito processes, and for each $A^{i}$ we require that

$$
\pi_{s} A_{s}^{i}=\mathbb{E}^{\mathbb{P}}\left[\pi_{t} A_{t}^{i} \mid \mathcal{F}_{s}\right]
$$

for $s \leq t$. Such an asset is characterized by its value $A_{T}^{i}$ at some terminal date $T$. In some situations it is useful to regard the asset as offering a single payment at $T$. In that case 
$\left\{A_{t}^{i}\right\}_{0 \leq t<T}$ represents the price process of the asset that offers such a payment. In other situations we can consider $A_{T}$ as being a "snapshot" of the value of the asset at time $T$. Usually the context will make it clear which meaning is intended.

Let $\left\{A_{t}^{0}\right\}$ be a money-market account in the base currency, initialised to unity. If we set $\rho_{t}=\pi_{t} A_{t}^{0}$ for $t \geq 0$, it follows that $\left\{\rho_{t}\right\}$ is a $\mathbb{P}$-martingale. A standard argument shows that $\left\{\rho_{t}\right\}$ can be used to make a change of measure. The resulting measure $\mathbb{Q}^{0}$ is the "riskneutral" measure associated with the base currency, and has the property that if the price of any non-dividend-paying asset is expressed in units of the money-market account, then the resulting process is a $\mathbb{Q}^{0}$-martingale. Thus, for each $i$ we have

$$
A_{s}^{i}=A_{s}^{0} \mathbb{E}^{\mathbb{Q}^{0}}\left[\frac{A_{t}^{i}}{A_{t}^{0}} \mid \mathcal{F}_{s}\right] .
$$

A similar situation arises with other choices of numeraire. Specifically, for any nondividend-paying asset $A^{i}$ of limited liability $\left(A_{t}^{i}>0\right)$, with price $\left\{A_{t}^{i}\right\}$, there is an associated measure $\mathbb{Q}^{i}$ with the property that if the price of any non-dividend-paying asset is expressed in units of $A^{i}$ then the result is a $\mathbb{Q}^{i}$-martingale. Thus for $0 \leq s \leq t$ and for all $i, j$ for which the price of $A^{i}$ is strictly positive we have:

$$
A_{s}^{j}=A_{s}^{i} \mathbb{E}^{\mathbb{Q}^{i}}\left[\frac{A_{t}^{j}}{A_{t}^{i}} \mid \mathcal{F}_{s}\right] .
$$

Bearing these points in mind, we observe that the option pricing problem can be formulated in the following context. We consider European-style options of a "Margrabe" type, for a pair of non-dividend-paying assets $A^{i}$ and $A^{j}$, where the option-holder has the right at time $t$ to exchange $K$ units of $A^{i}$ for one unit of $A^{j}$. The payoff $H_{t}^{i j}$ of such an option, in units of the base currency, is of the form

$$
H_{t}^{i j}(K)=\left(A_{t}^{j}-K A_{t}^{i}\right)^{+}
$$

The value of the option at $s \leq t$, expressed in units of the base currency, is given by

$$
C_{s t}^{i j}(K)=\frac{1}{\pi_{s}} \mathbb{E}^{\mathbb{P}}\left[\pi_{t}\left(A_{t}^{j}-K A_{t}^{i}\right)^{+} \mid \mathcal{F}_{s}\right]
$$

If $A^{i}$ is of limited liability, then the option value, expressed in units of $A^{i}$, is a $\mathbb{Q}^{i}$-martingale:

$$
\frac{C_{s t}^{i j}(K)}{A_{s}^{i}}=\mathbb{E}^{\mathbb{Q}^{i}}\left[\left(\frac{A_{t}^{j}}{A_{t}^{i}}-K\right)^{+} \mid \mathcal{F}_{s}\right] .
$$

This relation can be expressed more compactly as follows. Write $A_{s}$ for the price at time $s \leq t$ of a "generic asset" expressed in units of a "generic numeraire", and $C_{s t}(K)$ for the price, in units of the chosen numeraire, at time $s \leq t$, of a $t$-maturity $K$-strike option. Then the option payoff is given (in numeraire units) by

$$
H_{t}(K)=\left(A_{t}-K\right)^{+}
$$


The value of the option at time $s \leq t$ is

$$
C_{s t}(K)=\mathbb{E}^{\mathbb{Q}}\left[\left(A_{t}-K\right)^{+} \mid \mathcal{F}_{s}\right],
$$

where $\mathbb{Q}$ is the martingale measure associated with the numeraire. By "generic" we mean any choice of a non-dividend-paying assets $A^{i}$ and $A^{j}$ such that $A^{i}$ is of limited liability.

Standard options are not included in the category discussed above. A standard call has the payoff $H_{t}=\left(A_{t}-K\right)^{+}$where $A_{t}$ is the price of the underlying at time $t$ in currency units, and $K$ is a fixed strike in currency units. This is not an option to exchange $K$ units of a non-dividend-paying asset for one unit of another non-dividend-paying asset. One should think of the fixed strike as being $K$ units of a unit floating rate note. The only asset that maintains a constant value (in base-currency units) is a floating rate note - and such an asset pays a dividend. The dividend is the interest rate. In a given currency, by a floating rate note we mean an idealised note that pays interest continuously (rather than in lumps). The associated dividend is the short rate. One might argue that the strike on a standard option is cash, and that cash is a non-dividend-paying asset: this point of view leads to paradoxes. In the standard theory we regard cash as paying an implicit dividend, a convenience yield, in the form of a liquidity benefit equivalent to the interest rate. In summary, a standard option is an option to exchange the asset with a floating-rate note, which pays a "dividend".

Thus a standard option is a complicated entity - it is an option to exchange a certain number of units of a dividend-paying asset for one unit of a non-dividend-paying asset. It is more logical first to examine an option based on a pair of non-dividend-paying assets. In the literature this approach is implicitly adopted through the device of "setting the interest rate equal to zero". In that situation the floating rate note is non-dividend-paying; thus, the setting we operate within includes the zero-interest case. One would like to tackle the general problem of an option to exchange $K$ units of one dividend-paying asset for one unit of another dividend-paying asset (a "standard" foreign exchange option falls into that category); but, unless the dividend (or the interest rate) systems are deterministic, this is a more difficult problem than the one we propose to consider here.

The structure of the paper is as follows. In Section 2 we derive a dynamical equation for the conditional density, which we call the "master equation", given by (2.13). An integral form of the equation is presented in (2.14), which forms the basis of the solutions presented in later sections. In Section 3 we specify the general form we require the volatility structure of the conditional density to take, and give a characterization of what we mean by a "conditional density model" for asset pricing. In Section 4 we consider in detail the class of models for which the volatility structure of the conditional density is a deterministic function of two variables. This family of models admits a complete solution by use of a filtering technique. The resulting asset prices exhibit a stochastic volatility that is adapted to the market filtration but that is not in general of the local-volatility type. In Section 5 we consider the case when the volatility structure is linear in the terminal value of the asset. In that case the resulting models are Markovian, and can be calibrated to an arbitrary initial density. In Section 6 an alternative representation of the semi-linear case is presented using a Brownian-bridge technique. In Section 7 we show that the Bachelier model and the geometric Brownian motion model arise as special cases of the semilinear models, for particular choices of the initial density. We conclude in Section 8 with the calculation of option prices. 


\section{Conditional density processes}

The market is understood as having the setup described in the previous section. We have a probability space $(\Omega, \mathcal{F}, \mathbb{Q})$ with filtration $\left\{\mathcal{F}_{t}\right\}$. A non-dividend-paying limited-liability asset is chosen as numeraire, and all prices are expressed in units of that numeraire. The measure $\mathbb{Q}$ has the property that the price process of a non-dividend-paying asset, when expressed in units of the numeraire, is a martingale. We refer to $\mathbb{Q}$ as the martingale measure associated with this numeraire.

We fix $T>0$, and assume for $0 \leq t<T$ the existence of an $\mathcal{F}_{t^{-}}$conditional $\mathbb{Q}$-density $f_{t}(x)$ for $A_{T}$. In our applications we have in mind the cases $x \in \mathbb{R}$ and $x \in \mathbb{R}^{+}$, but it is useful to be flexible as regards the choice of the domain of the density function. In what follows we treat the case $x \in \mathbb{R}$, and leave it to the reader to supply the necessary adjustments for other domains. Thus we assume the existence of a density process $\left\{f_{t}(x)\right\}, x \in \mathbb{R}, 0 \leq t<T$, such that (1.3) holds. It follows that for any bounded, measurable function $g(x), x \in \mathbb{R}$, we have

$$
\int_{\mathbb{R}} g(x) f_{t}(x) \mathrm{d} x=\mathbb{E}\left[g\left(A_{T}\right) \mid \mathcal{F}_{t}\right]
$$

We ask that $\left\{f_{t}(x)\right\}$ should have those properties that follow heuristically as consequence of the formula obtained by formally differentiating (1.3), namely:

$$
f_{t}(x)=\mathbb{E}\left[\delta\left(x-A_{T}\right) \mid \mathcal{F}_{t}\right] .
$$

In particular, we require the following: (a) that for each $x \in \mathbb{R}$ the process $\left\{f_{t}(x)\right\}_{0 \leq t<T}$ is an $\left\{\mathcal{F}_{t}\right\}$-martingale, and hence $f_{s}(x)=\mathbb{E}\left[f_{t}(x) \mid \mathcal{F}_{s}\right]$ for $0 \leq s \leq t<T$; (b) that the price of the asset can be expressed in terms of the density by (1.4) for $t<T$; and (c) that

$$
\lim _{t \rightarrow T} \int_{\mathbb{R}} x f_{t}(x) \mathrm{d} x=A_{T} .
$$

We also assume, where required, that expressions analogous to (1.4) can be written for claims based on $A_{T}$. For example, if $C_{t T}(K)$ denotes the price at $t$ of a $T$-maturity, $K$-strike European call option, then we assume that (1.5) holds.

In the applications that follow, we introduce a $\mathbb{Q}$-Brownian motion $\left\{W_{t}\right\}$, which we take to be adapted to $\left\{\mathcal{F}_{t}\right\}$, and specialize to the case for which the dynamical equation of $\left\{f_{t}(x)\right\}$ is of the form

$$
f_{t}(x)=f_{0}(x)+\int_{0}^{t} \sigma_{s}^{f}(x) f_{s}(x) \mathrm{d} W_{s}
$$

for some process $\left\{\sigma_{t}^{f}(x)\right\}, x \in \mathbb{R}, 0 \leq t<T$, representing the volatility of the density. It follows that

$$
A_{t}=A_{0}+\int_{0}^{t} \sigma_{s}^{A} \mathrm{~d} W_{s}
$$

where $A_{0}=\int_{\mathbb{R}} x f_{0}(x) \mathrm{d} x$, and where the volatility of $A_{t}$ is given by

$$
\sigma_{t}^{A}=\int_{\mathbb{R}} x \sigma_{t}^{f}(x) f_{t}(x) \mathrm{d} x .
$$

For simplicity, we consider in this paper the case where $\left\{W_{t}\right\}$ is a one-dimensional Brownian motion. The extension to the multi-dimensional situation is unproblematic. 
Lemma 2.1. The normalisation condition

$$
\int_{\mathbb{R}} f_{t}(x) \mathrm{d} x=1
$$

holds for all $t \in[0, T)$ if and only if there exists a process $\left\{\sigma_{t}(x)\right\}$ such that

$$
\sigma_{t}^{f}(x)=\sigma_{t}(x) \int_{\mathbb{R}} f_{t}(y) \mathrm{d} y-\int_{\mathbb{R}} \sigma_{t}(y) f_{t}(y) \mathrm{d} y
$$

for almost all $t \in[0, T)$, and we have the initial condition

$$
\int_{\mathbb{R}} f_{0}(x) \mathrm{d} x=1
$$

Proof. First we show that (2.8) and (2.9) imply (2.7). Starting with (2.4), we integrate with respect to $x$ to obtain

$$
\int_{\mathbb{R}} f_{t}(x) \mathrm{d} x=\int_{\mathbb{R}} f_{0}(x) \mathrm{d} x+\int_{0}^{t} \int_{\mathbb{R}} \sigma_{s}^{f}(x) f_{s}(x) \mathrm{d} x \mathrm{~d} W_{s} .
$$

Inserting (2.8) and using (2.9) we obtain (2.7). Conversely, if we assume (2.7) then (2.9) holds as well, and hence

$$
\int_{0}^{t} \int_{\mathbb{R}} \sigma_{s}^{f}(x) f_{s}(x) \mathrm{d} x \mathrm{~d} W_{s}=0
$$

from which it follows that

$$
\int_{\mathbb{R}} \sigma_{t}^{f}(x) f_{t}(x) \mathrm{d} x=0
$$

for almost all $t \in[0, T)$, and thus that (2.8) holds for almost all $t \in[0, T)$ for some $\sigma_{t}(x)$.

Thus, once we specify $\left\{\sigma_{t}(x)\right\}$ and $\left\{f_{0}(x)\right\}$, the dynamical equation for the density - the so-called master equation-takes the form

$$
f_{t}(x)=f_{0}(x)+\int_{0}^{t}\left[\sigma_{s}(x)-\int_{\mathbb{R}} \sigma_{s}(y) f_{s}(y) \mathrm{d} y\right] f_{s}(x) \mathrm{d} W_{s} .
$$

Lemma 2.2. The conditional desity process $\left\{f_{t}(x)\right\}$ satisfies the master equation (2.13) with initial density $\left\{f_{0}(x)\right\}$ and volatility structure $\left\{\sigma_{t}(x)\right\}$ if and only if

$$
f_{t}(x)=\frac{f_{0}(x) \exp \left(\int_{0}^{t} \sigma_{s}(x) \mathrm{d} Z_{s}-\frac{1}{2} \int_{0}^{t} \sigma_{s}^{2}(x) \mathrm{d} s\right)}{\int_{\mathbb{R}} f_{0}(y) \exp \left(\int_{0}^{t} \sigma_{s}(y) \mathrm{d} Z_{s}-\frac{1}{2} \int_{0}^{t} \sigma_{s}^{2}(y) \mathrm{d} s\right) \mathrm{d} y},
$$

where

$$
Z_{t}=W_{t}+\int_{0}^{t} \int_{\mathbb{R}} \sigma_{s}(y) f_{s}(y) \mathrm{d} y \mathrm{~d} s .
$$


Proof. Writing (2.13) in differential form, we have

$$
\mathrm{d} f_{t}(x)=f_{t}(x)\left(\sigma_{t}(x)-\left\langle\sigma_{t}\right\rangle\right) \mathrm{d} W_{t},
$$

where for convenience we write

$$
\left\langle\sigma_{t}\right\rangle=\int_{\mathbb{R}} \sigma_{t}(x) f_{t}(x) \mathrm{d} x
$$

for the conditional "mean" of the volatility. We integrate (2.16) to obtain

$$
f_{t}(x)=f_{0}(x) \exp \left[\int_{0}^{t}\left(\sigma_{s}(x)-\left\langle\sigma_{s}\right\rangle\right) \mathrm{d} W_{s}-\frac{1}{2} \int_{0}^{t}\left(\sigma_{s}(x)-\left\langle\sigma_{s}\right\rangle\right)^{2} \mathrm{~d} s\right] .
$$

Expanding the exponent, we have

$$
f_{t}(x)=f_{0}(x) \frac{\exp \left[\int_{0}^{t} \sigma_{s}(x)\left(\mathrm{d} W_{s}+\left\langle\sigma_{s}\right\rangle \mathrm{d} s\right)-\frac{1}{2} \int_{0}^{t} \sigma_{s}^{2}(x) \mathrm{d} s\right]}{\exp \left[\int_{0}^{t}\left\langle\sigma_{s}\right\rangle\left(\mathrm{d} W_{s}+\left\langle\sigma_{s}\right\rangle \mathrm{d} s\right)-\frac{1}{2} \int_{0}^{t}\left\langle\sigma_{s}\right\rangle^{2} \mathrm{~d} s\right]} .
$$

Then we introduce a process $\left\{Z_{t}\right\}$ by writing

$$
Z_{t}=W_{t}+\int_{0}^{t}\left\langle\sigma_{s}\right\rangle \mathrm{d} s
$$

and it follows that

$$
f_{t}(x)=f_{0}(x) \frac{\exp \left[\int_{0}^{t} \sigma_{s}(x) \mathrm{d} Z_{s}-\frac{1}{2} \int_{0}^{t} \sigma_{s}^{2}(x) \mathrm{d} s\right]}{\exp \left[\int_{0}^{t}\left\langle\sigma_{s}\right\rangle \mathrm{d} Z_{s}-\frac{1}{2} \int_{0}^{t}\left\langle\sigma_{s}\right\rangle^{2} \mathrm{~d} s\right]} .
$$

Applying the normalization condition (2.7) to equation (2.21) above, we see that

$$
\begin{aligned}
& \exp \left(\int_{0}^{t}\left\langle\sigma_{s}\right\rangle \mathrm{d} Z_{s}-\frac{1}{2} \int_{0}^{t}\left\langle\sigma_{s}\right\rangle^{2} \mathrm{~d} s\right) \\
&=\int_{\mathbb{R}} f_{0}(x) \exp \left(\int_{0}^{t} \sigma_{s}(x) \mathrm{d} Z_{s}-\frac{1}{2} \int_{0}^{t} \sigma_{s}^{2}(x) \mathrm{d} s\right) \mathrm{d} x .
\end{aligned}
$$

It follows that (2.21) reduces to (2.14). Conversely, if $f_{t}(x)$ is given by (2.14), then it is a straightforward exercise in Ito calculus to check that the master equation is satisfied.

\section{Conditional density models}

We are in a position now to say more precisely what we mean by a "conditional density model". In doing so, we are motivated in part by advances in the study of infinite-dimensional stochastic differential equations. By a "model" for the density process we understand the following. We consider solutions of the master equation (2.13) satisfying the normalization 
condition (2.7), in conjunction with the specification of: (a) the initial density $f_{0}(x)$; and (b) the volatility structure $\left\{\sigma_{t}(x)\right\}$ in the form of a functional

$$
\sigma_{t}(x)=\Phi\left[f_{t}(\cdot), t, x\right]
$$

For each $x$ and $t$ the volatility $\sigma_{t}(x)$ depends on $f_{t}(y)$ for all $y \in \mathbb{R}$. Hence (2.13), thus specified, determines the dynamics of an infinite-dimensional Markov process.

The initial density $f_{0}(x)$ can be determined if one supplies initial option price data for the maturity date $T$ and for all strikes $K \in \mathbb{R}$. In particular, we have

$$
C_{0 T}(K)=\mathbb{E}\left[\left(A_{T}-K\right)^{+}\right]=\int_{\mathbb{R}}(x-K)^{+} f_{0}(x) \mathrm{d} x .
$$

By use of the idea of Breeden \& Litzenberger (1978) we see, in the present context, that for each value of $x$ one has

$$
f_{0}(x)=\frac{\partial^{2} C_{0 T}(x)}{\partial x^{2}}
$$

Here $f_{0}(x)$ is not generally the risk-neutral density, but rather the $\mathbb{Q}$-density of the value at time $T$ of the asset in units of the chosen numeraire. For simple practical applications, we take - in common with much of the literature - the numeraire to the be the money-market account, and set the interest rate to zero. Then $f_{0}(x)$ is the risk-neutral density. Once $f_{0}(x)$ has been supplied, the choice of the functional $\Phi$ determines the model for the conditional density: we give some examples later in the paper.

In practice, one would like to specify $\Phi$ modulo sufficient parametric freedom to allow the input of additional option price data. What form this additional data might take depends on the nature of the market and the class of valuation problems being pursued. For example, a standard problem would be to look at a limited-liability asset and consider additional data in the form of initial option prices for all strikes in $\mathbb{R}^{+}$and all maturities in the strip $0<t \leq T$. We require that $\Phi$ should be specified in such a way that once the data are provided, then $\Phi$ is determined and the "master equation" provides an evolution of the conditional density. Once we have the conditional density process, we can work out the evolution of the option price system for the specified strip, and hence the evolution of the associated implied volatility surface.

The data do not have to be presented exactly in the way specified in the previous paragraph - there may be situations where more data are available (e.g., in the form of barrier option prices or other derivative prices) or where less data are available (less welldeveloped markets). One should think of the parametric form of $\Phi$ as being adapted in a flexible way to the nature of a specific problem. The philosophy is that there are many different markets for options, and one needs a methodology that can accommodate these with reasonable generality.

It should be evident that an arbitrary solution to the master equation need not be a density process for an $\mathcal{F}_{t}$-measurable random variable - additional assumptions are required concerning the nature of the volatility structure in order to ensure that $f_{t}(x)$ converges in an appropriate sense to a suitable Dirac distribution. In the examples given, we indicate how this can be achieved, in various situations, by the choice of the volatility structure. 


\section{Models with deterministic volatility structures}

We proceed to present a rather general class of conditional density models characterized by a deterministic volatility structure. These are models for which $\left\{\sigma_{t}(x)\right\}$ is of the form

$$
\sigma_{t}(x)=v(t, x)
$$

for some deterministic function $v(t, x)$ defined for appropriate values of $t$ and $x$. We are able to give a more or less complete construction of such models in the form of a "weak" solution of the master equation. By a weak solution we mean that on a probability space $(\Omega, \mathcal{F}, \mathbb{Q})$ we construct a filtration $\left\{\mathcal{F}_{t}\right\}$, a Brownian motion $\left\{W_{t}\right\}$, and a conditional density process $\left\{f_{t}(x)\right\}$ satisfying the master equation and having the desired properties. More specifically, let $T \in(0, \infty)$ be fixed, and let $f_{0}(x): \mathbb{R} \rightarrow \mathbb{R}^{+}$be a prescribed initial density. Let $v(t, x)$ be a function on $[0, T) \times \mathbb{R}$ satisfying

$$
\int_{0}^{t} v(s, x)^{2} \mathrm{~d} s<\infty
$$

for $t<T$ and $x \in \mathbb{R}$, and let $\gamma(t)$ be a function on $[0, T)$ such that

$$
\lim _{t \rightarrow T} \gamma(t)=0, \quad \lim _{t \rightarrow T} \gamma(t) \int_{0}^{t} v(s, x) \mathrm{d} s=g(x),
$$

for some invertible function $g(x)$ on $\mathbb{R}$. We have the following:

Proposition 4.1. Let $X$ have density $f_{0}(x)$, and let $\left\{B_{t}\right\}$ be an independent Brownian motion. Let $\left\{\mathcal{F}_{t}\right\}$ be the filtration generated by the "information" process $\left\{I_{t}\right\}$ defined by

$$
I_{t}=B_{t}+\int_{0}^{t} v(s, X) \mathrm{d} s
$$

Let $\left\{f_{t}(x)\right\}$ be defined by

$$
f_{t}(x)=\frac{f_{0}(x) \exp \left[\int_{0}^{t} v(s, x) \mathrm{d} I_{s}-\frac{1}{2} \int_{0}^{t} v^{2}(s, x) \mathrm{d} s\right]}{\int_{-\infty}^{\infty} f_{0}(y) \exp \left[\int_{0}^{t} v(s, y) \mathrm{d} I_{s}-\frac{1}{2} \int_{0}^{t} v^{2}(s, y) \mathrm{d} s\right] \mathrm{d} y}
$$

and define $\left\{W_{t}\right\}$ by setting

$$
W_{t}=I_{t}-\int_{0}^{t} \mathbb{E}^{\mathbb{Q}}\left[v(s, X) \mid \mathcal{F}_{s}\right] \mathrm{d} s .
$$

Then: (a) the random variable $X$ is $\mathcal{F}_{T}$-measurable, and $f_{t}(x)$ is the associated conditional density; (b) the process $\left\{W_{t}\right\}$ is an $\left\{\mathcal{F}_{t}\right\}$-adapted Brownian motion; and (c) for $t \in[0, T)$, the density process $\left\{f_{t}(x)\right\}$ satisfies the master equation

$$
f_{t}(x)=f_{0}(x)+\int_{0}^{t} f_{s}(x)\left[v(s, x)-\int_{-\infty}^{\infty} v(s, y) f_{s}(y) \mathrm{d} y\right] \mathrm{d} W_{s} .
$$


Proof. It follows from (4.3) that $g(X)=\lim _{t \rightarrow T} \gamma(t) I_{t}$ is $\mathcal{F}_{T}$-measurable. Since $g(x)$ is invertible, we conclude that $X$ is $\mathcal{F}_{T}$-measurable. Now let the filtration $\left\{\mathcal{G}_{t}\right\}$ be defined by

$$
\mathcal{G}_{t}=\sigma\left(\left\{B_{s}\right\}_{0 \leq s \leq t}, X\right) .
$$

Clearly $\mathcal{F}_{t} \subset \mathcal{G}_{t}$. The random variable $X$ is $\mathcal{G}_{t}$-measurable, and $\left\{B_{t}\right\}$ is a $\left(\left\{\mathcal{G}_{t}\right\}, \mathbb{Q}\right)$-Brownian motion. We introduce a $\left(\left\{\mathcal{G}_{t}\right\}, \mathbb{Q}\right)$-martingale $\left\{M_{t}\right\}$ by setting

$$
M_{t}=\exp \left[-\int_{0}^{t} v(s, X) \mathrm{d} B_{s}-\frac{1}{2} \int_{0}^{t} v^{2}(s, X) \mathrm{d} s\right]
$$

for $t \in[0, T)$, and we let the probability measure $\mathbb{B}$ be defined by

$$
\left.\frac{\mathrm{d} \mathbb{B}}{\mathrm{d} \mathbb{Q}}\right|_{\mathcal{G}_{t}}=M_{t} .
$$

We observe that $\mathrm{d} I_{t}=\mathrm{d} B_{t}+v(t, X) \mathrm{d} t$. By Girsanov's theorem, $\left\{I_{t}\right\}$ is a $\left(\left\{\mathcal{G}_{t}\right\}, \mathbb{B}\right)$-Brownian motion. We note that $\left\{M_{t}^{-1}\right\}$ is a $\left(\left\{\mathcal{G}_{t}\right\}, \mathbb{B}\right)$-martingale. Let $H$ be a bounded measurable function on $\mathbb{R}$. Since $H(X)$ is $\mathcal{G}_{t}$-measurable, we have the generalised Bayes formula

$$
\mathbb{E}^{\mathbb{Q}}\left[H(X) \mid \mathcal{F}_{t}\right]=\frac{\mathbb{E}^{\mathbb{B}}\left[M_{t}^{-1} H(X) \mid \mathcal{F}_{t}\right]}{\mathbb{E}^{\mathbb{B}}\left[M_{t}^{-1} \mid \mathcal{F}_{t}\right]}
$$

Let us work out the right-hand side of this equation. To this end we show that $X$ and $I_{t}$ are $\mathbb{B}$-independent for all $t$. In particular, we show that the generating function

$$
\mathbb{E}^{\mathbb{B}}\left[\exp \left(y I_{t}+z X\right)\right]
$$

factorises. We have:

$$
\begin{aligned}
& \mathbb{E}^{\mathbb{B}}\left[\exp \left(y I_{t}+z X\right)\right]= \mathbb{E}^{\mathbb{Q}}\left[M_{t} \exp \left(y I_{t}+z X\right)\right], \\
&=\mathbb{E}^{\mathbb{Q}}\left[\operatorname { e x p } \left(-\int_{0}^{t} v(s, X) \mathrm{d} B_{s}-\frac{1}{2} \int_{0}^{t} v^{2}(s, X) \mathrm{d} s\right.\right. \\
&\left.\left.\quad+y\left[B_{t}+\int_{0}^{t} v(s, X) \mathrm{d} s\right]\right) \exp (z X)\right], \\
&=\mathbb{E}^{\mathbb{Q}}\left[m_{t} \exp \left(\frac{1}{2} y^{2} t\right) \exp (z X)\right],
\end{aligned}
$$

where

$$
m_{t}=\exp \left(\int_{0}^{t}[-v(s, X)+y] \mathrm{d} B_{s}-\frac{1}{2} \int_{0}^{t}[-v(s, X)+y]^{2} \mathrm{~d} s\right) .
$$

By use of the tower property and the independence of $\left\{B_{t}\right\}$ and $X$ under $\mathbb{Q}$, we have

$$
\begin{aligned}
\mathbb{E}^{\mathbb{B}}\left[\exp \left(y I_{t}+z X\right)\right] & =\mathbb{E}^{\mathbb{Q}}\left[m_{t} \exp \left(\frac{1}{2} y^{2} t\right) \exp (z X)\right] \\
& =\exp \left(\frac{1}{2} y^{2} t\right) \mathbb{E}^{\mathbb{Q}}\left[\mathbb{E}^{\mathbb{Q}}\left[m_{t} \exp (z X) \mid X\right]\right] \\
& =\exp \left(\frac{1}{2} y^{2} t\right) \mathbb{E}^{\mathbb{Q}}\left[\mathbb{E}^{\mathbb{Q}}\left[m_{t} \mid X\right] \mathbb{E}^{\mathbb{Q}}[\exp (z X)]\right]
\end{aligned}
$$


One observes that $\mathbb{E}^{\mathbb{Q}}\left[m_{t} \mid X\right]=1$. Thus we obtain the desired factorization:

$$
\mathbb{E}^{\mathbb{B}}\left[\exp \left(y I_{t}+z X\right)\right]=\exp \left(\frac{1}{2} y^{2} t\right) \mathbb{E}^{\mathbb{Q}}[\exp (z X)] .
$$

Now that we have shown that $X$ is $\mathbb{B}$-independent of $I_{t}$ (and thus of $\mathcal{F}_{t}$ ), we can work out the right-hand side of (4.11). We have:

$$
\begin{aligned}
\mathbb{E}^{\mathbb{Q}}\left[H(X) \mid \mathcal{F}_{t}\right] & =\frac{\mathbb{E}^{\mathbb{B}}\left[M_{t}^{-1} H(X) \mid \mathcal{F}_{t}\right]}{\mathbb{E}^{\mathbb{B}}\left[M_{t}^{-1} \mid \mathcal{F}_{t}\right]}, \\
& =\frac{\mathbb{E}^{\mathbb{B}}\left[H(X) \exp \left(\int_{0}^{t} v(s, X) \mathrm{d} I_{s}-\frac{1}{2} \int_{0}^{t} v^{2}(s, X) \mathrm{d} s\right) \mid \mathcal{F}_{t}\right]}{\mathbb{E}^{\mathbb{B}}\left[\exp \left(\int_{0}^{t} v(s, X) \mathrm{d} I_{s}-\frac{1}{2} \int_{0}^{t} v^{2}(s, X) \mathrm{d} s\right) \mid \mathcal{F}_{t}\right]}, \\
& =\frac{\int_{-\infty}^{\infty} f_{0}(x) H(x) \exp \left(\int_{0}^{t} v(s, x) \mathrm{d} I_{s}-\frac{1}{2} \int_{0}^{t} v^{2}(s, x) \mathrm{d} s\right) \mathrm{d} x}{\int_{-\infty}^{\infty} f_{0}(y) \exp \left(\int_{0}^{t} v(s, y) \mathrm{d} I_{s}-\frac{1}{2} \int_{0}^{t} v^{2}(s, y) \mathrm{d} s\right) \mathrm{d} y} .
\end{aligned}
$$

In particular, setting $H(X)=\mathbf{1}(X \leq x)$, we deduce that $f_{t}(x)$ is the $\mathcal{F}_{t}$-conditional density of $X$, as required. That proves the first part of the proposition.

Next we show that $\left\{W_{t}\right\}$ is an $\left(\left\{\mathcal{F}_{t}\right\}, \mathbb{Q}\right)$-Brownian motion. We need to show (1) that $\left(\mathrm{d} W_{t}\right)^{2}=\mathrm{d} t$, and $(2)$ that $\mathbb{E}^{\mathbb{Q}}\left[W_{u} \mid \mathcal{F}_{t}\right]=W_{t}$ for $0 \leq t \leq u$. The first condition is evidently satisfied. The second condition can be shown to be satisfied as follows. For simplicity, we suppress the superscript $\mathbb{Q}$. We have

$$
\mathbb{E}\left[W_{u} \mid \mathcal{F}_{t}\right]=\mathbb{E}\left[I_{u} \mid \mathcal{F}_{t}\right]-\mathbb{E}\left[\int_{0}^{u} \mathbb{E}\left[v(s, X) \mid \mathcal{F}_{s}\right] \mathrm{d} s \mid \mathcal{F}_{t}\right] .
$$

First we work out $\mathbb{E}\left[I_{u} \mid \mathcal{F}_{t}\right]$. Since $\left\{B_{t}\right\}$ is a $\left(\left\{\mathcal{G}_{t}\right\}, \mathbb{Q}\right)$-Brownian motion, we have

$$
\begin{aligned}
\mathbb{E}\left[I_{u} \mid \mathcal{F}_{t}\right] & =\mathbb{E}\left[B_{u}+\int_{0}^{u} v(s, X) \mathrm{d} s \mid \mathcal{F}_{t}\right] \\
& =\mathbb{E}\left[B_{u} \mid \mathcal{F}_{t}\right]+\mathbb{E}\left[\int_{0}^{u} v(s, X) \mathrm{d} s \mid \mathcal{F}_{t}\right] \\
& =\mathbb{E}\left[\mathbb{E}\left[B_{u} \mid \mathcal{G}_{t}\right] \mid \mathcal{F}_{t}\right]+\mathbb{E}\left[\int_{0}^{u} v(s, X) \mathrm{d} s \mid \mathcal{F}_{t}\right] \\
& =\mathbb{E}\left[B_{t} \mid \mathcal{F}_{t}\right]+\mathbb{E}\left[\int_{0}^{u} v(s, X) \mathrm{d} s \mid \mathcal{F}_{t}\right] .
\end{aligned}
$$

We insert this intermediate result in (4.24) to obtain

$$
\begin{aligned}
\mathbb{E}\left[W_{u} \mid \mathcal{F}_{t}\right] & =\mathbb{E}\left[I_{u} \mid \mathcal{F}_{t}\right]-\mathbb{E}\left[\int_{0}^{u} \mathbb{E}\left[v(s, X) \mid \mathcal{F}_{s}\right] \mathrm{d} s \mid \mathcal{F}_{t}\right] \\
& =\mathbb{E}\left[B_{t} \mid \mathcal{F}_{t}\right]+\mathbb{E}\left[\int_{0}^{u} v(s, X) \mathrm{d} s \mid \mathcal{F}_{t}\right]-\mathbb{E}\left[\int_{0}^{u} \mathbb{E}\left[v(s, X) \mid \mathcal{F}_{s}\right] \mathrm{d} s \mid \mathcal{F}_{t}\right] .
\end{aligned}
$$


Next we split the integrals in the last two expectations by writing,

$$
\begin{aligned}
\mathbb{E}\left[W_{u} \mid \mathcal{F}_{t}\right] & =\mathbb{E}\left[B_{t} \mid \mathcal{F}_{t}\right]+\mathbb{E}\left[\int_{0}^{t} v(s, X) \mathrm{d} s \mid \mathcal{F}_{t}\right]+\mathbb{E}\left[\int_{t}^{u} v(s, X) \mathrm{d} s \mid \mathcal{F}_{t}\right] \\
& -\mathbb{E}\left[\int_{0}^{t} \mathbb{E}\left[v(s, X) \mid \mathcal{F}_{s}\right] \mathrm{d} s \mid \mathcal{F}_{t}\right]-\mathbb{E}\left[\int_{t}^{u} \mathbb{E}\left[v(s, X) \mid \mathcal{F}_{s}\right] \mathrm{d} s \mid \mathcal{F}_{t}\right] .
\end{aligned}
$$

Observing that

$$
\mathbb{E}\left[B_{t} \mid \mathcal{F}_{t}\right]+\mathbb{E}\left[\int_{0}^{t} v(s, X) \mathrm{d} s \mid \mathcal{F}_{t}\right]=I_{t}
$$

and that

$$
\mathbb{E}\left[\int_{t}^{u} v(s, X) \mathrm{d} s \mid \mathcal{F}_{t}\right]=\mathbb{E}\left[\int_{t}^{u} \mathbb{E}\left[v(s, X) \mid \mathcal{F}_{s}\right] \mathrm{d} s \mid \mathcal{F}_{t}\right],
$$

we see that the expectation $\mathbb{E}\left[W_{u} \mid \mathcal{F}_{t}\right]$ reduces to

$$
\mathbb{E}\left[W_{u} \mid \mathcal{F}_{t}\right]=I_{t}-\mathbb{E}\left[\int_{0}^{t} \mathbb{E}\left[v(s, X) \mid \mathcal{F}_{s}\right] \mathrm{d} s \mid \mathcal{F}_{t}\right]=I_{t}-\int_{0}^{t} \mathbb{E}\left[v(s, X) \mid \mathcal{F}_{s}\right] \mathrm{d} s=W_{t} .
$$

That shows that $\left\{W_{t}\right\}$ is an $\left\{\mathcal{F}_{t}\right\}$-Brownian motion. An application of Ito calculus shows that the density process (4.5) satisfies the SDE (4.7).

Remark 4.1. For simplicity we have presented Proposition 4.1 for the case of a onedimensional state space. It should be clear from the proof how the results carry over to conditional densities on higher-dimensional state spaces.

Remark 4.2. For simulations of the dynamics of the conditional density process, the following alternative representation for (4.5) may prove useful:

$$
f_{t}(x)=\frac{f_{0}(x) \exp \left(\int_{0}^{t}[v(s, x)-v(s, X)] \mathrm{d} B_{s}-\frac{1}{2} \int_{0}^{t}[v(s, x)-v(s, X)]^{2} \mathrm{~d} s\right)}{\int_{\mathbb{R}} f_{0}(y) \exp \left(\int_{0}^{t}[v(s, y)-v(s, X)] \mathrm{d} B_{s}-\frac{1}{2} \int_{0}^{t}[v(s, y)-v(s, X)]^{2} \mathrm{~d} s\right) \mathrm{d} y} .
$$

The unnormalised density - the numerator in (4.35) - is for all $x \in \mathbb{R}$ conditionally lognormal given $X$. The simulation of the density requires only the numerical implementation of the standard Brownian motion and of the random variable $X$.

Remark 4.3. The density models with deterministic volatility structure presented in Proposition 4.1 can be extended to a class of models that satisfy the following system:

Let $f_{0}(x): \mathbb{R} \rightarrow \mathbb{R}^{+}$be a density function. A filtered probability space $\left(\Omega, \mathcal{F},\left\{\mathcal{F}_{t}\right\}, \mathbb{Q}\right)$ can be constructed along with (i) an $\mathcal{F}_{\infty}$-measurable random variable $X$ with density $f_{0}(x)$, (ii) an $\left\{\mathcal{F}_{t}\right\}$-adapted density process $\left\{f_{t}(x)\right\}$, and (iii) an $\left\{\mathcal{F}_{t}\right\}$-adapted Brownian motion $\left\{W_{t}\right\}$, such that (a) for some function $\gamma(t)$ on $[0, \infty)$, (b) for some function $g(x)$ that is invertible onto $\mathbb{R}$, and (c) for some suitably integrable function $v(t, x)$ on $[0, \infty) \times \mathbb{R}$ with the properties

$$
\lim _{t \rightarrow \infty} \sqrt{t} \gamma(t)=0, \quad \lim _{t \rightarrow \infty} \gamma(t) \int_{0}^{t} v(s, x) \mathrm{d} s=g(x)
$$


the following relations hold for all $t \in[0, \infty)$. We have $\mathbb{Q}\left[X \in \mathrm{d} x \mid \mathcal{F}_{t}\right]=f_{t}(x) \mathrm{d} x$ and

$$
f_{t}(x)=f_{0}(x)+\int_{0}^{t} f_{s}(x)\left[v(s, x)-\int_{-\infty}^{\infty} v(s, y) f_{s}(y) \mathrm{d} y\right] \mathrm{d} W_{s} .
$$

Remark 4.4. The construction of conditional density models admits an interpretation as a kind of a filtering problem. The process (4.4) plays the role of an "observation process", and (4.6) has the interpretation of being an "innovation process". See [1], [15], [16].

Remark 4.5. It is reasonable on a heuristic basis to expect that the general deterministic volatility structure model can be calibrated to the specification of an essentially arbitrary volatility surface. In particular, the parametric freedom implicit in a deterministic volatility structure coincides with that of a volatility surface. The situation is rather similar to that of the relation arising in the Dupire (1994) model between the local volatility (which is determined by a deterministic function of two variables, one with the dimensionality of price and the other with that of time) and the initial volatility surface (which represents a two-parameter family of option prices, labelled by strike and maturity). The precise characterisation of such relations constitutes a non-trivial and important inverse problem.

Remark 4.6. In the general deterministic volatility structure model, the dynamics of the underlying asset price $\left\{A_{t}\right\}$ are of the form $\mathrm{d} A_{t}=V_{t} \mathrm{~d} W_{t}$, with

$$
V_{t}=\int_{\mathbb{R}} x v(t, x) f_{t}(x) \mathrm{d} x-\int_{\mathbb{R}} x f_{t}(x) \mathrm{d} x \int_{\mathbb{R}} v(t, x) f_{t}(x) \mathrm{d} x,
$$

where $f_{t}(x)$ is given by (4.5). Thus the absolute volatility $V_{t}$ at time $t$ takes the form of a conditional covariance between $X$ and $v(t, X)$. In general, $\left\{V_{t}\right\}$ is an $\left\{\mathcal{F}_{t}\right\}$-adapted stochastic volatility process that cannot be expressed in the form $\Sigma\left(t, A_{t}\right)$ for some function $\Sigma(t, x)$, and the dynamics do not constitute a simple diffusion of the Dupire (local volatility) type. In the "semilinear" case $v(t, x)=\sigma T x /(T-t)$, however, the associated information process is Markovian, and $V_{t}$ can indeed be expressed in the form $\Sigma\left(t, A_{t}\right)$.

\section{Semilinear volatility structure}

We consider in this section the case where $A_{T}$ has a prescribed unconditional density $f_{0 T}(x)$, and construct a family of conditional density processes $\left\{f_{t T}\right\}$ that solve the master equation (2.13) over the time interval $[0, T)$. The filtration with respect to which $\left\{f_{t T}\right\}$ is defined will be constructed as follows. We introduce a process $\left\{\xi_{t T}\right\}_{0 \leq t \leq T}$ given by $\xi_{t T}=\sigma A_{T} t+\beta_{t T}$, where $\sigma$ is a constant and $\left\{\beta_{t T}\right\}_{0 \leq t \leq T}$ is a standard Brownian bridge, taken to be independent from $A_{T}$. We assume that $\left\{\mathcal{F}_{t}\right\}$ is given by

$$
\mathcal{F}_{t}=\sigma\left(\left\{\xi_{s T}\right\}_{0 \leq s \leq t}\right) .
$$

Clearly $\left\{\xi_{t T}\right\}$ is $\left\{\mathcal{F}_{t}\right\}$-adapted, and $A_{T}$ is $\mathcal{F}_{T}$-measurable. It is shown in Brody et al. (2007, 2008) that $\left\{\xi_{t T}\right\}$ is an $\left\{\mathcal{F}_{t}\right\}$-Markov process (see also Rutkowski \& Yu 2007). 
Proposition 5.1. Let the initial density $f_{0 T}(x)$ be prescribed, and let the volatility structure be of the semilinear form

$$
\sigma_{t T}(x)=\sigma \frac{T}{T-t} x
$$

for $0 \leq t<T$, and let $\left\{\mathcal{F}_{t}\right\}$ be defined by (5.1). Then the process $\left\{W_{t}\right\}_{0 \leq t<T}$ defined by

$$
W_{t}=\xi_{t T}-\int_{0}^{t} \frac{1}{T-s}\left(\sigma T \mathbb{E}\left[A_{T} \mid \xi_{s T}\right]-\xi_{s T}\right) \mathrm{d} s
$$

is an $\left\{\mathcal{F}_{t}\right\}$-Brownian motion, and the process $\left\{f_{t T}(x)\right\}$, given by

$$
f_{t T}(x)=\frac{f_{0 T}(x) \exp \left[\frac{T}{T-t}\left(\sigma \xi_{t T} x-\frac{1}{2} \sigma^{2} x^{2} t\right)\right]}{\int_{-\infty}^{\infty} f_{0 T}(y) \exp \left[\frac{T}{T-t}\left(\sigma \xi_{t T} y-\frac{1}{2} \sigma^{2} y^{2} t\right)\right] \mathrm{d} y},
$$

satisfies the master equation (2.13) with the given initial condition.

Proof. The fact that $\left\{W_{t}\right\}_{0 \leq t<T}$ is an $\left\{\mathcal{F}_{t}\right\}$-Brownian motion is shown in Brody et al. (2007, 2008). We work out $\mathbb{E}\left[A_{T} \mid \xi_{t T}\right]$ by use of the Bayes formula,

$$
\mathbb{E}\left[A_{T} \mid \xi_{t T}\right]=\int_{\mathbb{R}} x f_{t T}(x) \mathrm{d} x
$$

where

$$
f_{t T}(x)=\frac{f_{0 T}(x) \rho\left(\xi_{t T} \mid A_{T}=x\right)}{\int_{\mathbb{R}} f_{0 T}(y) \rho\left(\xi_{t T} \mid A_{T}=y\right) \mathrm{d} y} .
$$

Here $\rho\left(\xi_{t T} \mid A_{T}=x\right)$ is the conditional density of $\xi_{t T}$ given the value of $A_{T}$. We observe that $\xi_{t T}$ is conditionally Gaussian:

$$
\rho\left(\xi_{t T} \mid A_{T}=x\right)=\sqrt{\frac{T}{2 \pi t(T-t)}} \exp \left[-\frac{1}{2} \frac{T}{t(T-t)}\left(\xi_{t T}-\sigma t x\right)^{2}\right] .
$$

Thus the density process is given by

$$
f_{t T}(x)=\frac{f_{0 T}(x) \exp \left[-\frac{1}{2} \frac{T}{t(T-t)}\left(\xi_{t T}-\sigma t x\right)^{2}\right]}{\int_{-\infty}^{\infty} f_{0 T}(y) \exp \left[-\frac{1}{2} \frac{T}{t(T-t)}\left(\xi_{t T}-\sigma t y\right)^{2}\right] \mathrm{d} y} .
$$

The last expression can be simplified after some rearrangement so as to take the form

$$
f_{t T}(x)=\frac{f_{0 T}(x) \exp \left[\frac{T}{T-t}\left(\sigma \xi_{t T} x-\frac{1}{2} \sigma^{2} x^{2} t\right)\right]}{\int_{-\infty}^{\infty} f_{0 T}(y) \exp \left[\frac{T}{T-t}\left(\sigma \xi_{t T} y-\frac{1}{2} \sigma^{2} y^{2} t\right)\right] \mathrm{d} y} .
$$

With this result at hand, we can write the process $\left\{W_{t}\right\}_{0 \leq t<T}$ in the form

$$
W_{t}=\xi_{t T}-\int_{0}^{t} \frac{1}{T-s}\left(\sigma T \int_{\mathbb{R}} x f_{s T}(x) \mathrm{d} x-\xi_{s T}\right) \mathrm{d} s
$$


We recall that the master equation (2.13) can be written as (2.14). We shall prove that (5.4) satisfies (2.13) by showing that (2.14) reduces to (5.4) if we insert (5.3) in (2.20) and choose the volatility structure to be (5.2). For the process $\left\{Z_{t}\right\}$ in (2.20) we obtain

$$
Z_{t}=\xi_{t T}+\int_{0}^{t} \frac{\xi_{s T}}{T-s} \mathrm{~d} s
$$

The next step is to insert $\left\{Z_{t}\right\}$ in the exponent

$$
\int_{0}^{t} \sigma_{s T}(x) \mathrm{d} Z_{s}-\frac{1}{2} \int_{0}^{t} \sigma_{s T}(x)^{2} \mathrm{~d} s
$$

appearing in equation (2.14). Expression (5.12) can be simplified by use of (15.2) to give

$$
\sigma T x \int_{0}^{t} \frac{1}{T-s}\left(\mathrm{~d} \xi_{s T}+\frac{\xi_{s T}}{T-s} \mathrm{~d} s\right)-\frac{1}{2}(\sigma T x)^{2} \int_{0}^{t} \frac{1}{(T-s)^{2}} \mathrm{~d} s=\frac{T}{T-t}\left(\sigma x \xi_{t T}-\frac{1}{2} \sigma^{2} x^{2} t\right) .
$$

To derive this result, we make use of the relation

$$
\int_{0}^{t} \frac{\mathrm{d} \xi_{s T}}{T-s}=\frac{\xi_{t T}}{T-t}-\int_{0}^{t} \frac{\xi_{s T}}{(T-s)^{2}} \mathrm{~d} s
$$

With equation (5.13) at hand, we see that (2.14) reduces to (5.4) if (5.2) holds.

\section{Semilinear volatility: Brownian motion approach}

We proceed to show how the models constructed in Section 5 are related to the density models with deterministic volatility structure treated in Section 4. In particular, we consider a deterministic semilinear volatility function of the form

$$
v(t, x)=\sigma \frac{T}{T-t} x,
$$

where $0 \leq t<T$. For this volatility function the process $\left\{I_{t}\right\}$ has the dynamics

$$
\mathrm{d} I_{t}=\sigma \frac{T}{T-t} X \mathrm{~d} t+\mathrm{d} B_{t}
$$

We are thus able to work out the exponent

$$
\int_{0}^{t} v(s, x) \mathrm{d} I_{s}-\frac{1}{2} \int_{0}^{t} v^{2}(s, x) \mathrm{d} s
$$

in equation (4.5) making use of (6.1) and (6.2). We have:

$$
\begin{aligned}
& \int_{0}^{t} v(s, x) \mathrm{d} I_{s}-\frac{1}{2} \int_{0}^{t} v^{2}(s, x) \mathrm{d} s \\
& =\frac{T}{T-t} \sigma x\left[(T-t) \int_{0}^{t} \frac{\mathrm{d} B_{s}}{T-s}+T(T-t) \sigma X \int_{0}^{t} \frac{\mathrm{d} s}{(T-s)^{2}}\right]-\frac{1}{2} T^{2} \sigma^{2} x^{2} \int_{0}^{t} \frac{\mathrm{d} s}{(T-s)^{2}} .
\end{aligned}
$$


The first integral gives rise to a $\left(\left\{\mathcal{G}_{t}\right\}, \mathbb{Q}\right)$-Brownian bridge $\left\{\beta_{t T}\right\}$ over the interval $[0, T]$. More specifically, we have

$$
\beta_{t T}=(T-t) \int_{0}^{t} \frac{\mathrm{d} B_{s}}{T-s}
$$

The deterministic integral in (6.4) gives

$$
\int_{0}^{t} \frac{\mathrm{d} s}{(T-s)^{2}}=\frac{t}{T(T-t)}
$$

Armed with these results, one can write (6.3) as follows:

$$
\int_{0}^{t} v(s, x) \mathrm{d} I_{s}-\frac{1}{2} \int_{0}^{t} v^{2}(s, x) \mathrm{d} s=\frac{T}{T-t} \sigma x\left(\sigma X t+\beta_{t T}\right)-\frac{1}{2} \frac{T}{T-t} \sigma^{2} x^{2} t .
$$

Let $\left\{\xi_{t T}\right\}$ be defined for $t \in[0, T]$ by $\xi_{t T}=\sigma X t+\beta_{t T}$. Then for (6.7) we obtain

$$
\int_{0}^{t} v(s, x) \mathrm{d} I_{s}-\frac{1}{2} \int_{0}^{t} v^{2}(s, x) \mathrm{d} s=\frac{T}{T-t}\left(\sigma x \xi_{t T}-\frac{1}{2} \sigma^{2} x^{2} t\right) .
$$

We conclude that the conditional density process $\left\{f_{t}(x)\right\}$ in (4.5) reduces to the following expression in the case for which the volatility structure is given by (6.1):

$$
f_{t}(x)=\frac{f_{0}(x) \exp \left[\frac{T}{T-t}\left(\sigma x \xi_{t T}-\frac{1}{2} \sigma^{2} x^{2} t\right)\right]}{\int_{-\infty}^{\infty} f_{0}(y) \exp \left[\frac{T}{T-t}\left(\sigma y \xi_{t T}-\frac{1}{2} \sigma^{2} y^{2} t\right)\right] \mathrm{d} y} .
$$

From equation (6.8) we see that $\left\{\xi_{t T}\right\}$ takes the role of the information process that generates $\left\{\mathcal{F}_{t}\right\}$. Since $\left\{\beta_{t T}\right\}$ vanishes for $t=T$, the random variable $X$ is "revealed" at $T$. Thus $X$ is $\mathcal{F}_{T}$-measurable, and $\left\{\xi_{t T}\right\}$ is the process generating the information-based models of Brody et al. $(2007,2008)$. This conclusion is supported by the following construction.

We consider the measure $\mathbb{B}$ defined in (4.10). Under $\mathbb{B}$ the process $\left\{I_{t}\right\}$ is a Brownian motion over the interval $[0, T)$. We construct a $\mathbb{B}$-Brownian bridge by use of the $\mathbb{B}$-Brownian motion $\left\{I_{t}\right\}$ as follows. On $[0, T)$ we set

$$
\xi_{t T}=(T-t) \int_{0}^{t} \frac{1}{T-s} \mathrm{~d} I_{s} .
$$

Next we recall definition (4.4) and insert this in the expression above. The result is

$$
\xi_{t T}=(T-t) \int_{0}^{t} \frac{\mathrm{d} B_{s}}{T-s}+(T-t) \int_{0}^{t} \frac{1}{T-s} v(s, X) \mathrm{d} s .
$$

The first integral defines a $\left(\left\{\mathcal{G}_{t}\right\}, \mathbb{Q}\right)$-Brownian bridge over the interval $[0, T)$ which we denote $\left\{\beta_{t T}\right\}$. For the volatility function we set

$$
v(t, x)=\sigma \frac{T}{T-t} x .
$$

This leads to

$$
\xi_{t T}=\sigma X(T-t) T \int_{0}^{t} \frac{1}{(T-s)^{2}} \mathrm{~d} s+\beta_{t T}
$$

and thence to (6.8). 


\section{Bachelier model}

The Bachelier model is obtained by setting $A_{t}=\gamma W_{t}$ where $\gamma$ is a constant. We shall show that the class of models defined by Proposition 5.1 contains the Bachelier model. We consider a random variable $A_{T}$ associated with a fixed date $T$. We assume that $A_{T} \sim N\left[0,1 /\left(T \sigma^{2}\right)\right]$, where $N[m, v]$ is the class of Gaussian random variables with mean $m$ and variance $v$. In the notation of Section 5 , we have

$$
f_{0 T}(x)=\frac{\sigma \sqrt{T}}{\sqrt{2 \pi}} \exp \left(-\frac{1}{2} \sigma^{2} T x^{2}\right) .
$$

We recall the process $\left\{\xi_{t T}\right\}_{0 \leq t \leq T}$ defined by $\xi_{t T}=\sigma A_{T} t+\beta_{t T}$. If $A_{T} \sim N\left(0,1 /\left(T \sigma^{2}\right)\right)$, then $\left\{\xi_{t T}\right\}$ is an $\left\{\mathcal{F}_{t}\right\}$-Brownian motion over $[0, T]$. This is because $\left\{\xi_{t T}\right\}$ is a continuous Gaussian process with $\xi_{0 T}=0$ and $\operatorname{Cov}\left[\xi_{s T}, \xi_{t T}\right]=s$ for $0 \leq s \leq t \leq T$. We recall the definition of the Brownian motion $\left\{W_{t}\right\}$ associated with $\left\{\xi_{t T}\right\}$, given by (5.3). Since for $A_{T} \sim N\left(0,1 / T \sigma^{2}\right)$ the process $\left\{\xi_{t T}\right\}$ is a Brownian motion, it follows that

$$
\mathbb{E}\left[A_{T} \mid \xi_{s T}\right]=\frac{1}{\sigma T} \mathbb{E}\left[\xi_{T T} \mid \xi_{s T}\right]=\frac{1}{\sigma T} \xi_{s T} .
$$

Thus we see that $W_{t}=\xi_{t T}$. As a consequence we have

$$
A_{t}=\mathbb{E}\left[A_{T} \mid \xi_{t T}\right]=\frac{1}{\sigma T} \mathbb{E}\left[W_{T} \mid W_{t}\right]=\frac{1}{\sigma T} W_{t} .
$$

Hence to match the Bachelier model with an element in the class of models constructed in Section 5, it suffices to set $\sigma=1 /(\gamma T)$.

Proposition 7.1. The conditional density process $\left\{f_{t T}^{B}(x)\right\}$ of the Bachelier price process, defined over the interval $[0, T)$, given by

$$
f_{t T}^{B}(x)=\frac{\exp \left[-\frac{1}{2} \frac{1}{\gamma^{2}(T-t)}\left(x-\gamma W_{t}\right)^{2}\right]}{\int_{-\infty}^{\infty} \exp \left[-\frac{1}{2} \frac{1}{\gamma^{2}(T-t)}\left(y-\gamma W_{t}\right)^{2}\right] \mathrm{d} y},
$$

is a special case of the family of the models of Proposition 5.1, and is obtained by setting

$$
f_{0 T}(x)=\frac{\sigma \sqrt{T}}{\sqrt{2 \pi}} \exp \left(-\frac{1}{2} \sigma^{2} T x^{2}\right), \quad \sigma_{t T}(x)=\sigma \frac{T}{T-t} x, \quad \text { and } \quad \sigma=1 /(\gamma T) .
$$

Proof. We insert (7.5) in (5.4). Completion of squares gives

$$
\begin{aligned}
f_{t T}(x) & =\frac{f_{0 T}(x) \exp \left[\frac{T}{T-t}\left(\sigma \xi_{t T} x-\frac{1}{2} \sigma^{2} x^{2} t\right)\right]}{\int_{-\infty}^{\infty} f_{0 T}(y) \exp \left[\frac{T}{T-t}\left(\sigma \xi_{t T} y-\frac{1}{2} \sigma^{2} y^{2} t\right)\right] \mathrm{d} y} \\
& =\frac{\exp \left[-\frac{1}{2} \frac{\sigma^{2} T^{2}}{T-t}\left(x-\frac{1}{\sigma T} \xi_{t T}\right)^{2}\right]}{\int_{-\infty}^{\infty} \exp \left[-\frac{1}{2} \frac{\sigma^{2} T^{2}}{T-t}\left(y-\frac{1}{\sigma T} \xi_{t T}\right)^{2}\right] \mathrm{d} y} .
\end{aligned}
$$

Recalling that $\xi_{t T}=W_{t}$, and setting $\sigma=1 /(\gamma T)$, we obtain the desired result. 
Remark 7.1. Let the initial density $f_{0 T}(x)$, the volatility structure $\sigma_{t T}(x)$, and the parameter $\sigma$ be given as in (7.5). Then the Bachelier conditional density $\left\{f_{t T}^{B}(x)\right\}$ satisfies the master equation (2.13), and $\left\{W_{t}\right\}$ coincides with $\left\{\xi_{t T}\right\}$.

Remark 7.2. Suppose we chose an asset price model with a certain law. Then we know that we can derive the corresponding conditional density process where the related volatility structure and initial density are specified. We may then wonder how the conditional density transforms, and what the new volatility structure looks like, if we consider a new law for the asset price model. For instance, we may begin with the Bachelier model and ask what is the conditional density and volatility structure associated with a log-normal model. We present a "transformation formula" for the conditional density. This result allows for the construction of a variety of conditional density processes from a given one. Let $\left\{f_{t}(x)\right\}$ solve (2.13), and let $\psi: \mathbb{R} \rightarrow \mathbb{R}$ be a $C^{1}$-bijection. Then it is known that if $X$ has conditional density $f_{t}(x)$ then $Z=\psi(X)$ has conditional density $g_{t}(z)$ given by

$$
g_{t}(z)=\frac{f_{t}\left(\psi^{-1}(z)\right)}{\psi^{\prime}\left(\psi^{-1}(z)\right)}
$$

where $\psi^{-1}(z)$ is the inverse function and $\psi^{\prime}(x)$ is the derivative of $\psi(x)$. The conditional density $\left\{g_{t}(z)\right\}$ satisfies

$$
\mathrm{d} g_{t}(z)=g_{t}(z)\left[\nu(t, z)-\left\langle\nu_{t}\right\rangle\right] \mathrm{d} W_{t},
$$

where $\nu(t, z)=v\left(t, \psi^{-1}(z)\right)$ and

$$
\left\langle\nu_{t}\right\rangle=\int_{\mathbb{R}} v(t, y) g_{t}(y) \mathrm{d} y
$$

We see that the volatility structure $v(t, x)$ associated with $f_{t}(x)$ becomes the volatility structure $\nu(t, z)$ associated with $g_{t}(z)$. For example, consider the Bachelier model where $A_{T} \sim N\left(0,1 /\left(T \sigma^{2}\right)\right)$. The associated initial density and volatility structure are given in (7.5). Now suppose that $Z=\exp \left(A_{T}\right)$, so $\psi(x)=\exp (x)$. The price process $\left\{A_{t}\right\}$ is then given by the log-normal model

$$
A_{t}=\exp \left(\gamma W_{t}\right)
$$

where $W_{t}=\xi_{t T}$. It follows by (7.7) that the conditional density process $\left\{g_{t T}(x)\right\}$ associated with the log-normal price process (7.10) is

$$
g_{t T}(z)=\frac{\exp \left[-\frac{1}{2} \frac{1}{\gamma^{2}(T-t)}\left(\ln (z)-\gamma W_{t}\right)^{2}\right]}{z \int_{0}^{\infty} \exp \left[-\frac{1}{2} \frac{1}{\gamma^{2}(T-t)}\left(\ln (y)-\gamma W_{t}\right)^{2}\right] \mathrm{d} y},
$$

for $z>0$. Indeed we see that $g_{t T}(z)$ is the log-normal conditional density. The associated volatility structure is

$$
\nu(t, z)=v(t, \ln (z))=\sigma_{t T}(z)=\sigma \frac{T}{T-t} \ln (z) .
$$




\section{Option prices}

We consider a European-style call option with maturity $t$, strike $K$, and price as determined by equation (1.13). The price process $\left\{A_{t}\right\}_{0 \leq t<\infty}$ of the underlying asset is given by

$$
A_{t}=\mathbb{E}^{\mathbb{Q}}\left[X \mid \mathcal{F}_{t}\right]=\frac{\int_{-\infty}^{\infty} x f_{0}(x) \exp \left(\int_{0}^{t} v(s, x) \mathrm{d} I_{s}-\frac{1}{2} \int_{0}^{t} v^{2}(s, x) \mathrm{d} s\right) \mathrm{d} x}{\int_{-\infty}^{\infty} f_{0}(y) \exp \left(\int_{0}^{t} v(s, y) \mathrm{d} I_{s}-\frac{1}{2} \int_{0}^{t} v^{2}(s, y) \mathrm{d} s\right) \mathrm{d} y}
$$

where $\left\{\mathcal{F}_{t}\right\}_{0 \leq s \leq t<\infty}$ is generated by (4.4). We recall that $\left\{B_{t}\right\}$ is a $\left(\left\{\mathcal{G}_{t}\right\}, \mathbb{Q}\right)$-Brownian motion, and that there exists an $\left(\left\{\mathcal{F}_{t}\right\}, \mathbb{Q}\right)$-Brownian motion $\left\{W_{t}\right\}$ such that

$$
\mathrm{d} I_{t}=\mathrm{d} W_{t}+\left\langle v_{t}\right\rangle \mathrm{d} t,
$$

where the bracket notation is defined by (2.17). We introduce a positive $\left(\left\{\mathcal{F}_{t}\right\}, \mathbb{Q}\right)$-martingale

$$
\Lambda_{t}=\exp \left(\int_{0}^{t}\left\langle v_{s}\right\rangle \mathrm{d} W_{s}+\frac{1}{2} \int_{0}^{t}\left\langle v_{s}\right\rangle^{2} \mathrm{~d} s\right)
$$

which induces a change of measure from $\mathbb{Q}$ to measure $\mathbb{Q}^{*}$ given by

$$
\left.\frac{\mathrm{d} \mathbb{Q}^{*}}{\mathrm{~d} \mathbb{Q}}\right|_{\mathcal{F}_{t}}=\Lambda_{t}
$$

The $\mathbb{Q}^{*}$-measure is characterised by the fact that $\left\{I_{t}\right\}$ is an $\left(\left\{\mathcal{F}_{t}\right\}, \mathbb{Q}^{*}\right)$-Brownian motion. We observe that by the relationship

$$
\begin{aligned}
\int_{\mathbb{R}} f_{0}(x) \exp \left(\int_{0}^{t} v(s, x) \mathrm{d} I_{s}\right. & \left.-\frac{1}{2} \int_{0}^{t} v^{2}(s, x) \mathrm{d} s\right) \mathrm{d} x \\
& =\exp \left(\int_{0}^{t}\left\langle v_{s}\right\rangle \mathrm{d} I_{s}-\frac{1}{2} \int_{0}^{t}\left\langle v_{s}\right\rangle^{2} \mathrm{~d} s\right) \\
& =\Lambda_{t},
\end{aligned}
$$

we can use the denominator in (8.1) to write the option price $C_{s t}$ in terms of a conditional expectation taken with respect to $\mathbb{Q}^{*}$ under which $\left\{I_{t}\right\}$ is a Brownian motion. Equation (8.6) is obtained by applying the relationship (8.2). We then have

$$
\begin{aligned}
C_{s t} & =\mathbb{E}^{\mathbb{Q}}\left[\left(A_{t}-K\right)^{+} \mid \mathcal{F}_{s}\right] \\
& =\mathbb{E}^{\mathbb{Q}}\left[\left(N_{t} \Lambda_{t}^{-1}-K\right)^{+} \mid \mathcal{F}_{s}\right] \\
& =\mathbb{E}^{\mathbb{Q}}\left[\Lambda_{t}^{-1}\left(N_{t}-K \Lambda_{t}\right)^{+} \mid \mathcal{F}_{s}\right] \\
& =\Lambda_{s}^{-1} \mathbb{E}^{\mathbb{Q}^{*}}\left[\left(N_{t}-K \Lambda_{t}\right)^{+} \mid \mathcal{F}_{s}\right],
\end{aligned}
$$

where

$$
\begin{aligned}
N_{t} & =\int_{-\infty}^{\infty} x f_{0}(x) \exp \left(\int_{0}^{t} v(s, x) \mathrm{d} I_{s}-\frac{1}{2} \int_{0}^{t} v^{2}(s, x) \mathrm{d} s\right) \mathrm{d} x \\
\Lambda_{t} & =\int_{-\infty}^{\infty} f_{0}(x) \exp \left(\int_{0}^{t} v(s, x) \mathrm{d} I_{s}-\frac{1}{2} \int_{0}^{t} v^{2}(s, x) \mathrm{d} s\right) \mathrm{d} x .
\end{aligned}
$$


Since $\left\{I_{t}\right\}$ is an $\left(\left\{\mathcal{F}_{t}\right\}, \mathbb{Q}^{*}\right)$-Brownian motion, the conditional expectation simplifies to the calculation of a Gaussian integral provided the zero of the max function can be computed.

With these formulae in hand, we observe that a closed-form expression for the conditional expectation can be worked out in the case of a binary initial density function of the form

$$
f_{0}(x)=q_{1} \delta\left(x-x_{1}\right)+q_{2} \delta\left(x-x_{2}\right) .
$$

Here $\delta(x)$ is the Dirac distribution and $q_{i}=\mathbb{Q}\left[X=x_{i}\right]$ for $i=1,2$. It follows that

$$
N_{t}=\sum_{i=1}^{2} x_{i} q_{i} \mathcal{E}_{t}\left(x_{i}\right), \quad \Lambda_{t}=\sum_{i=1}^{2} q_{i} \mathcal{E}_{t}\left(x_{i}\right)
$$

where we introduce the process

$$
\mathcal{E}_{t}\left(x_{i}\right)=\exp \left[\int_{0}^{t} v\left(s, x_{i}\right) \mathrm{d} I_{s}-\frac{1}{2} \int_{0}^{t} v^{2}\left(s, x_{i}\right) \mathrm{d} s\right] .
$$

In the case where the random variable $X$ takes the values $x_{1}$ and $x_{2}$, the option price is

$$
C_{s t}=\Lambda_{s}^{-1} \mathbb{E}_{s}^{\mathbb{Q}^{*}}\left[\left(\sum_{i=1}^{2}\left(x_{i}-K\right) q_{i} \mathcal{E}_{t}\left(x_{i}\right)\right)^{+} \mid \mathcal{F}_{s}\right]
$$

We observe that $\left\{\mathcal{E}\left(x_{i}\right)\right\}$ is positive and has the property $\mathbb{E}^{\mathbb{Q}^{*}}\left[\mathcal{E}_{t}\left(x_{i}\right)\right]=1$. In particular, we can use $\left\{\mathcal{E}_{t}\left(x_{1}\right)\right\}$ to define a change of measure from $\mathbb{Q}^{*}$ to a new measure $\tilde{\mathbb{Q}}$ by setting

$$
\left.\frac{\mathrm{d} \tilde{\mathbb{Q}}}{\mathrm{d} \mathbb{Q}^{*}}\right|_{\mathcal{F}_{t}}=\mathcal{E}_{t}\left(x_{1}\right)
$$

together with $\mathrm{d} I_{t}=\mathrm{d} \tilde{I}_{t}+v\left(t, x_{1}\right) \mathrm{d} t$. Next we pull $\mathcal{E}_{t}\left(x_{1}\right)$ out to the front of the max function in equation (8.16) to obtain

$$
C_{s t}=\Lambda_{s}^{-1} \mathbb{E}^{\mathbb{Q}^{*}}\left[\mathcal{E}_{t}\left(x_{1}\right)\left(q_{1}\left(x_{1}-K\right)+q_{2}\left(x_{2}-K\right) \frac{\mathcal{E}_{t}\left(x_{2}\right)}{\mathcal{E}_{t}\left(x_{1}\right)}\right)^{+} \mid \mathcal{F}_{s}\right] .
$$

By use of the Bayes formula we express the option price in terms of $\tilde{\mathbb{Q}}$ :

$$
C_{s t}=\Lambda_{s}^{-1} \mathcal{E}_{s}\left(x_{1}\right) \mathbb{E}^{\tilde{\mathbb{Q}}}\left[\left(q_{1}\left(x_{1}-K\right)+q_{2}\left(x_{2}-K\right) \frac{\mathcal{E}_{t}\left(x_{2}\right)}{\mathcal{E}_{t}\left(x_{1}\right)}\right)^{+} \mid \mathcal{F}_{s}\right] .
$$

For the sake of a simplified notation we define $R_{0 t}=\mathcal{E}_{t}\left(x_{2}\right) / \mathcal{E}_{t}\left(x_{1}\right)$, and we observe that the process $\left\{R_{0 t}\right\}$ is an exponential $\left(\left\{\mathcal{F}_{t}\right\}, \tilde{\mathbb{Q}}\right)$-martingale:

$$
R_{0 t}=\exp \left(\int_{0}^{t}\left[v\left(s, x_{2}\right)-v\left(s, x_{1}\right)\right] \mathrm{d} \tilde{I}_{s}-\frac{1}{2} \int_{0}^{t}\left[v\left(s, x_{2}\right)-v\left(s, x_{1}\right)\right]^{2} \mathrm{~d} s\right) .
$$

We write $R_{0 t}=R_{0 s} R_{s t}$ so that

$$
C_{s t}=\Lambda_{s}^{-1} \mathcal{E}_{s}\left(x_{1}\right) \mathbb{E}^{\tilde{\mathbb{Q}}}\left[\left(q_{1}\left(x_{1}-K\right)+q_{2}\left(x_{2}-K\right) R_{0 s} R_{s t}\right)^{+} \mid \mathcal{F}_{s}\right],
$$


and we note that $R_{0 s}$ is $\mathcal{F}_{s}$-measurable. One is thus left with the task of finding the range of values of $R_{s t}$ for which the max function vanishes. Then we calculate the Gaussian integral arising from the conditional expectation. Recalling that $\left\{\tilde{I}_{t}\right\}$ is an $\left(\left\{\mathcal{F}_{t}\right\}, \tilde{\mathbb{Q}}\right)$-Brownian motion, we note that the logarithm of $R_{s t}$ is Gaussian. Let $Y$ be a standard Gaussian variable. Then we can write

$$
\ln \left(R_{s t}\right)=\sqrt{\int_{s}^{t}\left[v\left(u, x_{2}\right)-v\left(u, x_{1}\right)\right]^{2} \mathrm{~d} u} Y-\frac{1}{2} \int_{s}^{t}\left[v\left(u, x_{2}\right)-v\left(u, x_{1}\right)\right]^{2} \mathrm{~d} u .
$$

By solving for the logarithm of $R_{s t}$ in the argument of the max function in (8.21), we deduce that the max function is zero for all values $y^{*}$ that $Y$ may take for which

$$
y^{*} \leq \frac{\ln \left[\frac{q_{1}\left(K-x_{1}\right)}{q_{2}\left(K-x_{2}\right) R_{0 s}}\right]+\frac{1}{2} \int_{s}^{t}\left[v\left(u, x_{2}\right)-v\left(u, x_{1}\right)\right]^{2} \mathrm{~d} u}{\sqrt{\int_{s}^{t}\left[v\left(u, x_{2}\right)-v\left(u, x_{1}\right)\right]^{2} \mathrm{~d} u}} .
$$

It follows therefore that the option price can be written in the form

$$
\begin{aligned}
C_{s t}=\Lambda_{s}^{-1} \mathcal{E}_{s}\left(x_{1}\right)\left[q_{1}\left(x_{1}-K\right)\right. & \frac{1}{\sqrt{2 \pi}} \int_{y^{*}}^{\infty} \exp \left(-\frac{1}{2} y^{2}\right) \mathrm{d} y \\
& \left.+q_{2}\left(x_{2}-K\right) R_{0 s} \frac{1}{\sqrt{2 \pi}} \int_{y^{*}}^{\infty} \exp \left(-\frac{1}{2} \eta^{2}(y)\right) \mathrm{d} y\right]
\end{aligned}
$$

where

$$
\eta(y)=y-\sqrt{\int_{s}^{t}\left[v\left(u, x_{2}\right)-v\left(u, x_{1}\right)\right]^{2} \mathrm{~d} u} .
$$

The two Gaussian integrals can be written in terms of the normal distribution function $N(x)$. To highlight the similarity with the Black-Scholes option price formula, we define

$$
d_{s t}^{-}=-y^{*}, \quad d_{s t}^{+}=d_{s t}^{-}+\sqrt{\int_{s}^{t}\left[v\left(u, x_{2}\right)-v\left(u, x_{1}\right)\right]^{2} \mathrm{~d} u}
$$

so that one can write

$$
C_{s t}=\Lambda_{s}^{-1} \mathcal{E}_{s}\left(x_{1}\right)\left[q_{1}\left(x_{1}-K\right) N\left(d_{s t}^{-}\right)+q_{2}\left(x_{2}-K\right) R_{0 s} N\left(d_{s t}^{+}\right)\right] .
$$

We can simplify this expression further by use of (8.14). Finally, we conclude that the price of the call option is given by the following compact formula:

$$
C_{s t}=\left(x_{1}-K\right) \frac{q_{1}}{q_{1}+q_{2} R_{0 s}} N\left(d_{s t}^{-}\right)+\left(x_{2}-K\right) \frac{q_{2}}{q_{1} R_{0 s}^{-1}+q_{2}} N\left(d_{s t}^{+}\right) .
$$


Acknowledgements. The authors thank T. Björk, Y. Kabanov, M. Monoiyos, M. Schweizer, J. Sekine, J. Zubelli, and participants at the Sixth World Congress of the Bachelier Finance Society for useful discussions. Part of this research has been carried out as part of the project "Dynamic Asset Pricing", National Centre of Competence in Research "Financial Valuation and Risk Management" (NCCR FINRISK), a research instrument of the Swiss National Science Foundation. LPH and AM are grateful to the Ludwig-Maximilians-Universität München and the Fields Institute, Toronto, for hospitality. LPH acknowledges support from Lloyds TSB Bank Plc, Kyoto University, Shell UK Ltd, and the Aspen Center for Physics. AM acknowledges support by ESF through AMaMeF grant 2863, and thanks the Vienna Institute of Finance and EPFL for the stimulating work environment. Part of this research was carried out while AM was a member of the Department of Mathematics, ETH Zürich.

\section{References.}

1. Bensoussan, A. (1992) Stochastic Control of Partially Observable Systems. Cambridge University Press.

2. Breeden, D. T. \& Litzenberger, R.H. (1978) Prices of state-contingent claims implicit in option prices. Journal of Business 51, 621-651.

3. Brody, D. C. \& Hughston, L. P. (2001a) Interest rates and information geometry. Proceedings of the Royal Society A 457, 1343-1364.

4. Brody, D. C. \& Hughston, L. P. (2001b) Applications of information geometry to interest rate theory. Disordered and Complex Systems, eds. P. Sollich, A. C. C. Coolen, L. P. Hughston \& R. F. Streater (New York: AIP).

5. Brody, D. C. \& Hughston, L. P. (2002) Entropy and information in the interest rate term structure. Quantitative Finance 2, 70-80.

6. Brody, D. C., Hughston, L. P. \& Macrina, A. (2007) Beyond hazard rates: a new approach to credit risk modelling. In Advances in Mathematical Finance, Festschrift volume in honour of Dilip Madan. R. Elliott, M. Fu, R. Jarrow \& Ju-Yi Yen eds., Birkhäuser.

7. Brody, D. C., Hughston, L. P. \& Macrina, A. (2008) Information-based asset pricing. International Journal of Theoretical and Applied Finance 11, No. 1, 107-142.

8. Carmona, R. \& Nadtochiy, S. (2009) Local volatility dynamic models. Finance and Stochastics 13, 1-48.

9. Carmona, R. \& Nadtochiy, S. (2011) Tangent models as a mathematical framework for dynamic calibration. International Journal of Theoretical and Applied Finance 14, No. 1, 107-135.

10. Davis, M. (2004) Complete-market models of stochastic volatility. Proceedings of the Royal Society London A 460, 11-26.

11. Dupire, B. (1994) Pricing with a smile. Risk 7, 18-20. 
12. El Karoui, N., Jeanblanc, M. \& Jiao, Y. (2010) What happens after a default: the conditional density approach. Stochastic Processes and their Applications 120, No. 7, 1011-1032.

13. Filipović, D., Tappe, S. \& Teichmann, J. (2010) Term structure models driven by Wiener process and Poisson measures: existence and positivity. SIAM Journal on Financial Mathematics 1, 523-554.

14. Gatheral, J. (2006) The Implied Volatility Surface: a Practitioner's Guide. Wiley.

15. Krylov, N. V. (1980) Controlled Diffusion Processes. Springer.

16. Liptser, R. S. \& Shiryaev, A. N. (2001) Statistics of Random Processes, I. General Theory, and II. Applications. 2nd edition, Springer.

17. Rutkowski, M. \& Yu, N. (2007) An extension of the Brody-Hughston-Macrina approach to modeling of defaultable bonds. International Journal of Theoretical and Applied Finance 10, No. 3, 557-589.

18. Schönbucher, P. J. (1999) A market model for stochastic implied volatility. Philosophical Transactions of the Royal Society A 357, 2071-2092.

19. Schweizer, M. \& Wissel, J. (2008a) Term structure of implied volatilities: absence of arbitrage and existence results. Mathematical Finance 18, No. 1, 77-114.

20. Schweizer, M. \& Wissel, J. (2008b) Arbitrage-free market models for option prices: the multi-strike case. Finance and Stochastics 12, 469-505. 